\title{
A systematic review of the association between delayed appropriate therapy and mortality among patients hospitalized with infections due to Klebsiella pneumoniae or Escherichia coli: how long is too long?
}

\author{
Thomas P. Lodise', Qi Zhao ${ }^{2}$, Kyle Fahrbach ${ }^{3}$, Patrick J. Gillard ${ }^{4 *}$ and Amber Martin ${ }^{3}$
}

\begin{abstract}
Background: Temporal relationships between the time to appropriate antibiotic therapy and outcomes are not well described.

Methods: A systematic literature review and meta-analysis was performed to examine this relationship in patients hospitalized with Klebsiella pneumoniae or Escherichia coli infections.

Results: Twenty identified studies contained data for patients who received delayed appropriate therapy (DAT) versus appropriate antibiotic therapy for these pathogens. Of the 20 included studies, the majority (19/20) focused on patients with bloodstream infections, and only 1 study evaluated patients with pneumonia. When all DAT results were combined (any delay $>24 \mathrm{~h}$ from culture collection or any delay after culture and susceptibility reporting [C\& SR]), there was an increased risk of mortality (odds ratio [OR], 1.60 [95\% Cl, 1.25-2.50]). The risk of mortality was greater when DAT > $48 \mathrm{~h}$ from culture collection or DAT > C\&SR results were combined (OR, $1.76[95 \% \mathrm{Cl}, 1.27-2.44])$.
\end{abstract}

Conclusions: Our findings suggest there is a need to shift current treatment practices away from antibiotic escalation strategies that contribute to delayed appropriate therapy and toward early, relatively aggressive and comprehensive, antibiotic therapy, especially among patients with bloodstream infections due to K. pneumoniae or E. coli.

Keywords: Escherichia coli, Gram-negative infection, Klebsiella pneumoniae, Mortality, Delayed treatment

\section{Background}

Recent data from the Study for Monitoring Antimicrobial Resistance Trends program [1] found that during 2011 the global rates of extended-spectrum beta lactamase (ESBL)containing Escherichia coli from intra-abdominal infections ranged from 6\% (North America) to 38\% (Asia). During the preceding decade, rates for urinary tract infections (UTIs) caused by ESBL E. coli and Klebsiella pneumoniae increased from 3.3 to $8.0 \%$ and from 9.1 to $18.6 \%$, respectively, across 217 hospitals in the United States [2]. Results from the Healthcare Cost and

\footnotetext{
* Correspondence: gillard_patrick@allergan.com

${ }^{4}$ Global Health Economics and Outcomes Research, Allergan plc, 2525

Dupont Drive, Irvine, CA 92612, USA

Full list of author information is available at the end of the article
}

Utilization Project Nationwide Inpatient Sample database [2] show that the number of complicated (ie, hospitalized) UTIs caused by carbapenem-resistant Enterobacteriaceae (CRE) in the United States increased from 0\% in 2000 to $2.3 \%$ in 2009. The clinical burden of these resistant infections is substantial: ESBL-producing Enterobacteriaceae and CRE account for an estimated 1700 and 610 deaths annually, respectively, in the United States [3].

Reasons for concern regarding the rising rates of resistance are many. In more than $25 \%$ of patients with resistant Gram-negative infections, initial empirical antibiotic therapy has no microbiological activity against the pathogen causing the infection $[4,5]$. Patients who receive delayed appropriate therapy (DAT) may have a

(c) The Author(s). 2018 Open Access This article is distributed under the terms of the Creative Commons Attribution 4.0 International License (http://creativecommons.org/licenses/by/4.0/), which permits unrestricted use, distribution, and reproduction in any medium, provided you give appropriate credit to the original author(s) and the source, provide a link to the Creative Commons license, and indicate if changes were made. The Creative Commons Public Domain Dedication waiver (http://creativecommons.org/publicdomain/zero/1.0/) applies to the data made available in this article, unless otherwise stated. 
1.5- to 2-fold increase in morbidity and mortality relative to those who receive early appropriate therapy [6].

A second reason for concern is that resistance limits treatment options. For ESBLs, clinicians are often left using last-line agents, such as carbapenems, empirically. Clinicians have had to rely on older, often toxic agents (eg, colistin) with limited efficacy data to treat infections caused by CRE $[7,8]$.

Although the deleterious outcomes associated with DAT are well described, the definitive time delay associated with worse outcomes has not been well quantified. There is no standardized definition of DAT; different definitions are used throughout the literature, with DAT variously defined as nonreceipt of microbiologically active treatment within 24,48 , or $72 \mathrm{~h}$ of culture collection or at the time of culture and susceptibility reporting (C\& SR). Thus, the question remains: how long a delay is too long? The intent of this study was to examine the association between delay in appropriate therapy and mortality in patients with infections due to K. pneumoniae or E. coli.

\section{Methods}

This review was conducted in accordance with the Preferred Reporting Items for Systematic Reviews and Meta-Analyses (PRISMA) guidelines.

\section{Search strategy}

Systematic searches were conducted in the MEDLINE (via PubMed), EMBASE, and Cochrane literature databases. Because the terms "inappropriate therapy" and "delayed appropriate therapy" are used interchangeably in the literature, both terms were paired with terms for Gram-negative pathogens and infection types of interest to identify English-language studies published between January 1, 2004, and June 13, 2014. Complete details of the search algorithms are provided in Additional file 1.

\section{Inclusion and exclusion criteria}

All identified abstracts were manually reviewed against the prespecified inclusion/exclusion criteria considering participants, interventions, comparisons, outcomes, study design, and time period (PICOS-T) elements. Included studies evaluated adults hospitalized with Gram-negative infections (ie, pneumonia, bloodstream infections, complicated UTIs, and complicated intra-abdominal infections) caused by $K$. pneumoniae or E. coli. Studies were required to have extractable outcomes data regarding DAT, which was defined as nonreceipt of microbiologically active treatment within 24,48 , or $72 \mathrm{~h}$ of culture collection (DAT $>24,>48$, or $>72 \mathrm{~h}$ ) or at the time of C\& SR (DAT $>$ C\& SR). Studies that involved pediatric patients; used treatments other than antibiotics; were animal, in vitro, or genetic; were narrative publications, nonsystematic reviews, case studies, case reports, or editorials; or were comparative studies with $<10$ patients per treatment group or single-arm studies with fewer than 20 patients were excluded. Also excluded were studies reporting $<5 \%$ of patients receiving DAT $>$ C\& SR compared with those who received appropriate antibiotics.

\section{Data extraction and statistical analysis}

Extraction of the included studies was performed using a Microsoft $\mathrm{Excel}^{\circ}$ extraction template. Meta-analysis was conducted using R version 2.15.2 [9]. Study-level data, patient characteristics, treatment details, and mortality were captured from full-text articles by a single investigator. All extracted data were validated by a second investigator to confirm the completeness and accuracy of extraction. The validated data were eligible for inclusion in statistical analysis. Random-effects meta-analyses of odds ratios (ORs) for mortality were conducted when data permitted for the following DAT definitions by K. pneumoniae and E. coli combined and by each organism individually:

- All DAT (includes studies with DAT $>24,>48$, or $>72 \mathrm{~h}$ from the time of culture collection and results from studies with DAT $>$ C\& SR)

- DAT $>24 h$ and DAT $>$ C\& SR, combined

- DAT $>48 \mathrm{~h}$ and DAT $>$ C\& SR, combined o This approximates the typical 48- to 72-h time frame for reporting of culture and susceptibility data

- Additional DAT categories o DAT $>24 \mathrm{~h}$ o DAT $>48 \mathrm{~h}$ o DAT $>72 \mathrm{~h}$ o DAT $>$ C\& SR

For clarity, studies that include a DAT $>24 \mathrm{~h}$ may include delays in starting appropriate therapy of $>48 \mathrm{~h}$; studies that include a DAT of $>48 \mathrm{~h}$ may include delays in starting appropriate therapy of $>72 \mathrm{~h}$ but will not include delays in starting therapy of $<48 \mathrm{~h}$.

The random-effects method considers any variation between studies as a random variable, enabling the analysis to account for any heterogeneity observed. Heterogeneity was quantified using classical meta-analysis methods (ie, Cochran $\mathrm{Q}$ and the $\mathrm{I}^{2}$ measure). Heterogeneity is defined as differences between study results beyond what would be expected from sampling error (ie, $\tau^{2}>0$ ) [10]. It may stem from several sources, including differences across studies in patient inclusion and exclusion criteria, differences in accuracy of laboratory measurement, and differences in reporting conventions (eg, group means at baseline vs sensitivity/ specificity). 


\section{Results}

\section{Systematic literature search}

The literature searches yielded 1844 unique articles after results from each database were merged and duplicate citations were removed. Of these, 1550 studies were rejected during abstract screening because they did not meet inclusion criteria.

Full-text articles of the remaining 294 citations were retrieved for further examination, during which 164 articles were rejected, most commonly because they did not report mortality for the population of interest (ie, patients infected with Klebsiella spp. or E. coli; $n=112$ ). Of the remaining 130 studies, 110 were excluded because they either did not report outcomes data for patients receiving DAT $(n=107)$ or they reported outcomes for patients who received DAT $>$ C\& $\mathrm{SR}$, the proportion of whom was $<5 \%$ of those patients who received appropriate antibiotics in the same study $(n=3)$. An overview of the reasons for study attrition is shown in Fig. 1. Of the 20 included studies, the majority $(19 / 20)$ focused on patients with bloodstream infections, and only 1 study evaluated patients with pneumonia [11]. Table 1 [11-30] summarizes the mortality results for the 20 included studies.
Patient mortality by delayed appropriate therapy time frame

\section{Overall delayed appropriate therapy}

When all DAT results were combined for meta-analysis, infection due to $K$. pneumoniae or E. coli was associated with a significantly higher risk of overall mortality (OR, 1.60 [95\% CI, 1.25-2.05]; Fig. 2a [11-30]). Heterogeneity was low $\left(I^{2}=32.4 \%, Q p=0.130\right)$, with the most apparent outliers seen in Tuon et al. [26] on the low end (OR= 0.67) and Lee et al. [30] and Schiappa et al. [25] on the high end ( $\mathrm{OR}=5.05$ and 12.86, respectively).

In the 9 studies reporting outcomes for patients with K. pneumoniae infections $[11,13,16,17,19,21,22,26$, $29]$, the overall DAT was associated with a significantly higher risk of overall mortality (OR, 1.53 [95\% CI, 1.072.17]; Fig. 2b [11, 13, 16, 17, 19, 21, 22, 26, 29]). With the exception of 3 studies [19, 26, 29], the proportion of patients who died was higher in those patient groups that received DAT compared with those receiving early appropriate antibiotics. Heterogeneity was low $\left(\mathrm{I}^{2}=\right.$ $25.2 \%, \mathrm{Qp}=0.226$ ), with the most extreme results being an OR of 0.67 on the lower end in Tuon et al. [26] and an OR of 3.79 on the high end in Anderson et al. [13].

Eight studies reported overall mortality data for patients with $E$. coli infection alone $[15,17,18,20,21,23,24,28]$. In

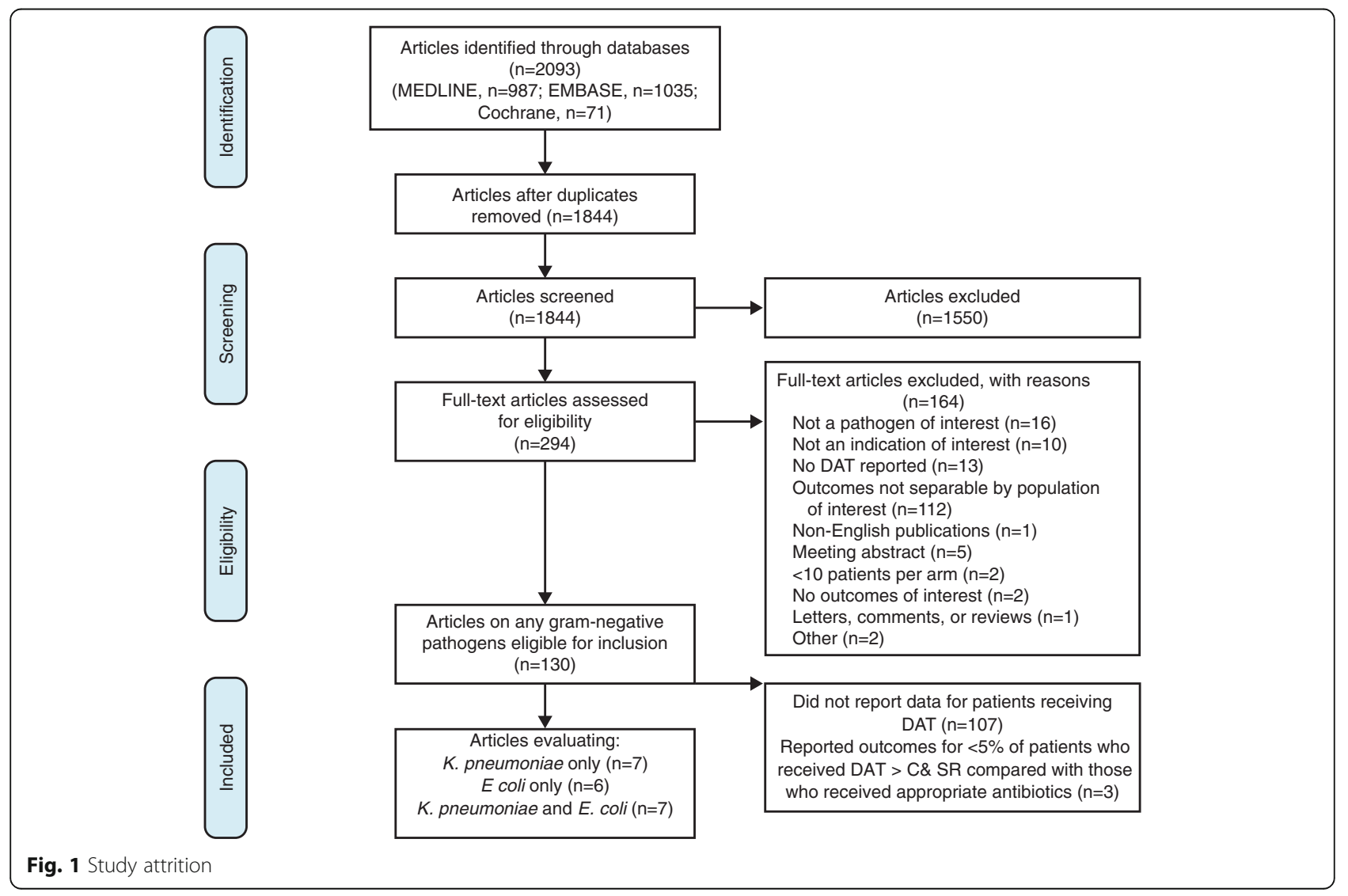




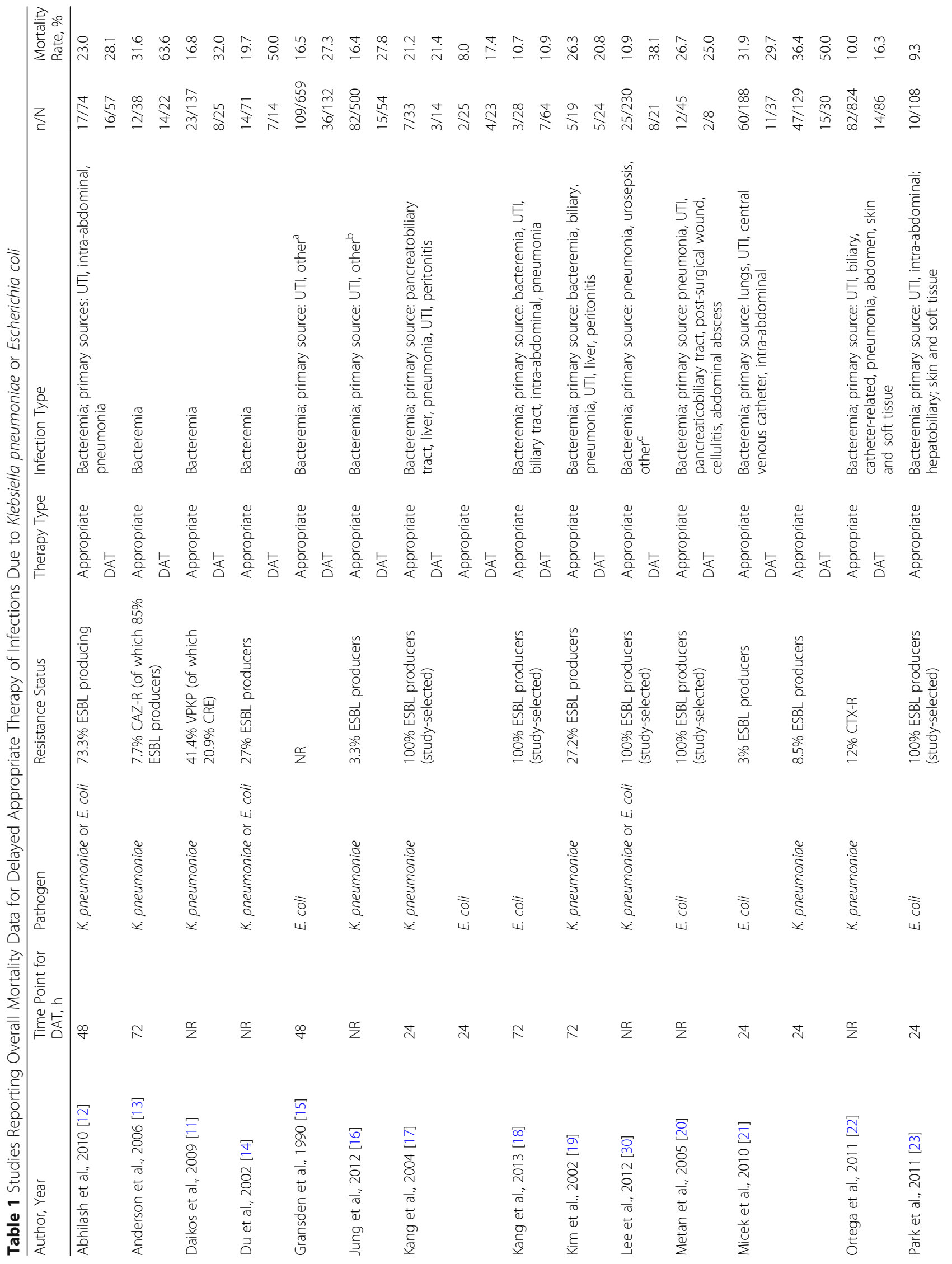




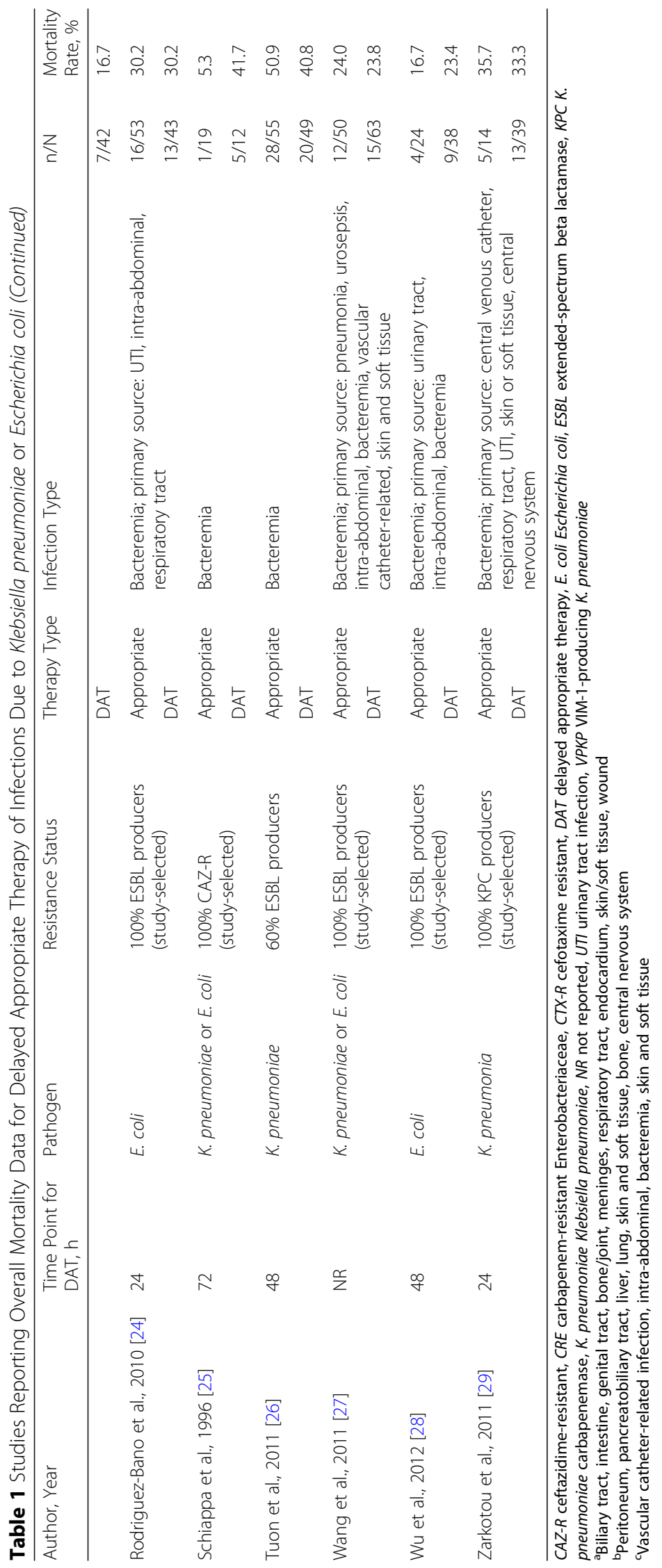




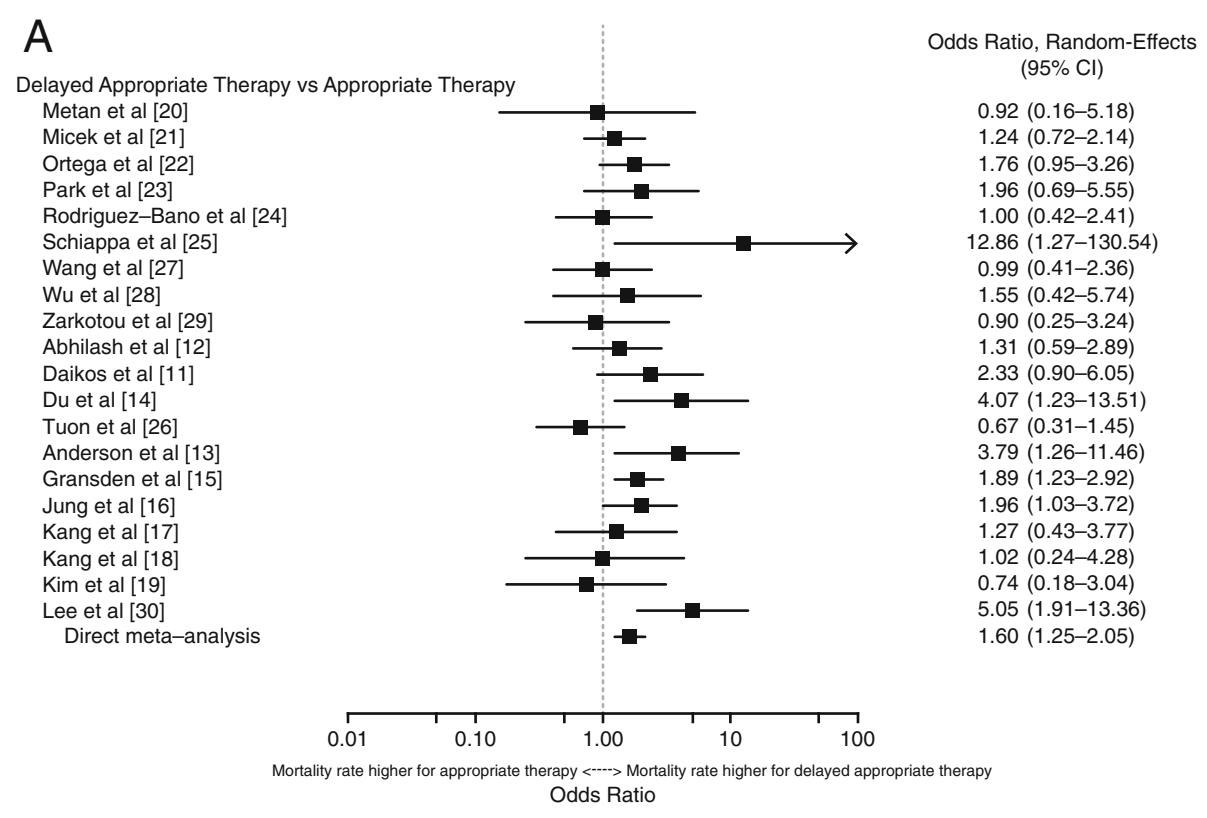

B

Delayed Appropriate Therapy vs Appropriate Therapy Anderson et al [13]

Daikos et al [11]

Tuon et al [26]

Jung et al [16]

Kang et al [17]

Kim et al [19]

Micek et al [21]

Ortega et al [22]

Zarkotou et al [29]

Direct meta-analysis

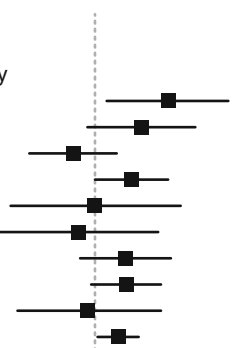

Odds Ratio, Random-Effects $(95 \% \mathrm{Cl})$

$3.79(1.26-11.46)$

$2.33(0.90-6.05)$

$0.67(0.31-1.45)$

$1.96(1.03-3.72)$

$1.01(0.22-4.66)$

$0.74(0.18-3.04)$

$1.74(0.78-3.88)$

$1.76(0.95-3.26)$

$0.90(0.25-3.24)$

$1.53(1.07-2.17)$

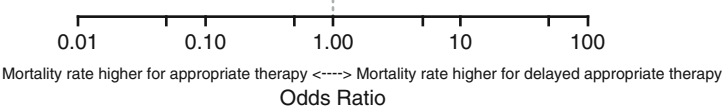

Odds Ratio

Overall Mortality (Combining Timepoints)

C

Delayed Appropriate Therapy vs Appropriate Therapy Gransden et al [15]

Kang et al [17]

Kang et al [18]

Metan et al [20]

Micek et al [21]

Park et al [23]

Rodriguez-Bano et al [24]

Wu et al [28]

Direct meta-analysis

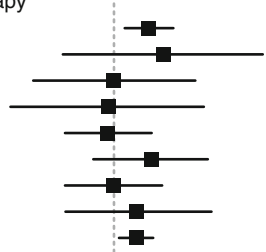

Odds Ratio, Random-Effects $(95 \% \mathrm{Cl})$

$1.89(1.23-2.92)$

$2.42(0.40-14.69)$

$1.02(0.24-4.28)$

$0.92(0.16-5.18)$

$0.90(0.42-1.95)$

$1.96(0.69-5.55)$

$1.00(0.42-2.41)$

$1.55(0.42-5.74)$

$1.49(1.10-2.01)$

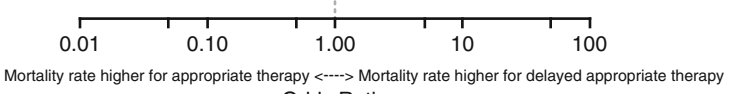

Odds Ratio

Fig. 2 Overall mortality for all studies with any DAT $>24 \mathrm{~h}$ or DAT $>$ C\& SR of infections due to (a) K. pneumoniae or E. coli, (b) K. pneumoniae alone, and (c) E. coli alone

these studies, DAT was similarly associated with a significantly higher risk of overall mortality (OR, $1.49[95 \%$ CI, 1.10-2.01]; Fig. 2c [15, 17, 18, 20, 21, 23, 24, 28]). There was no sign of heterogeneity in the results
$\left(\mathrm{I}^{2}=0.0 \%, \mathrm{Qp}=0.697\right)$. The proportion of patients who died was higher in those groups receiving DAT than in those who received appropriate therapy in 5 of the 8 studies (Table 1). 


\section{Delayed appropriate therapy $>48$ hours or delayed} appropriate therapy $>C \& S R$, combined

Meta-analysis results comparing overall mortality in patients with $K$. pneumoniae or $E$. coli infections who received DAT $>48 \mathrm{~h}$ or DAT $>\mathrm{C} \&$ SR combined $(n=15)$ are shown in Fig. 3a [11-16, 18-20, 22, 25-28, 30]. There was a statistically significant increase in the rate of mortality associated with combined results from DAT $>48$ h or DAT $>$ C\& SR (OR, 1.76 [95\% CI, $1.27-2.44])$. Heterogeneity was moderate $\left(\mathrm{I}^{2}=45.8 \%\right.$, $\mathrm{Qp}=0.070)$ and was driven by Tuon et al. [26] and Kim et al. [19] on the low end and Schiappa et al. [25] on the high end.

Three studies compared overall mortality in patients with infections due to $K$. pneumoniae who received DAT $>48 \mathrm{~h}[13,19,26]$, and another 3 studies reported results

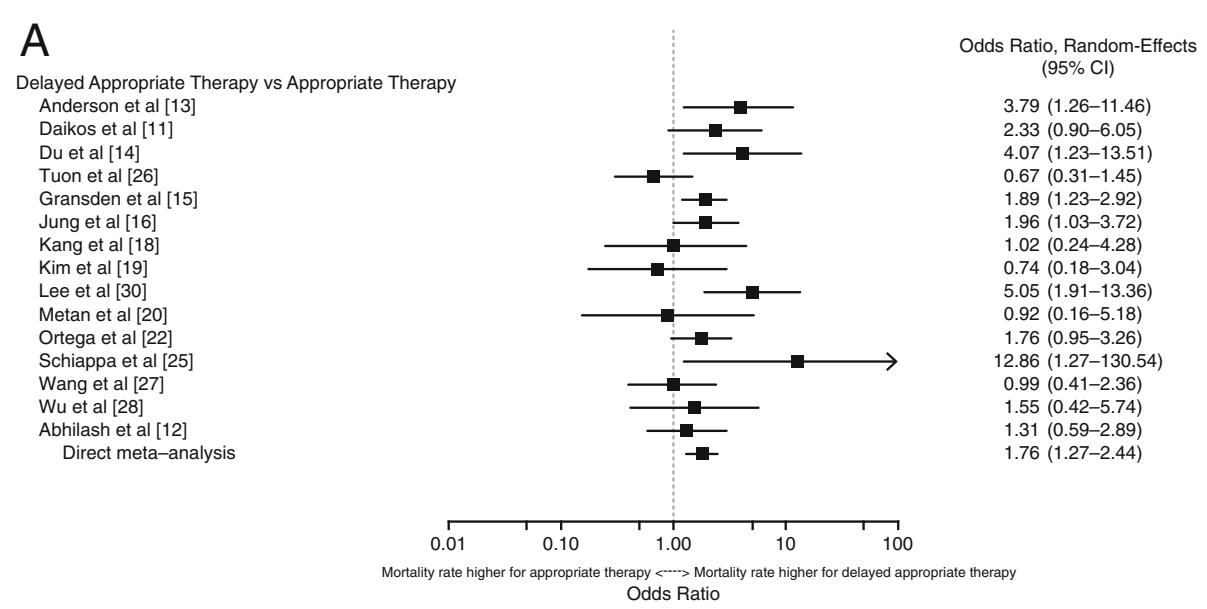

B Delayed Appropriate Therapy vs Appropriate Therapy Anderson et al [13] Daikos et al [11] Tuon et al [26] Jung et al [16] Kim et al [19] Kim et al [19]
Ortega et al [22] Direct meta-analysis

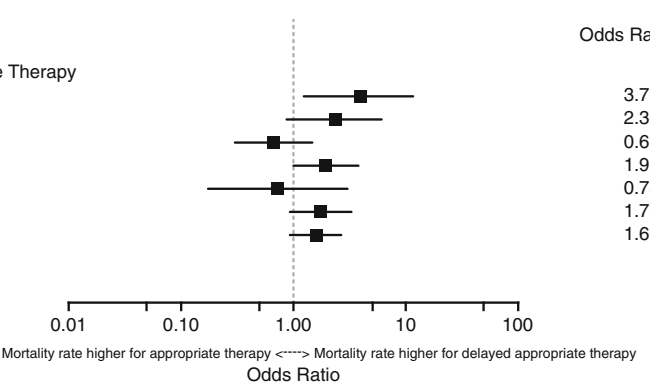

Odds Ratio, Random-Effects $(95 \% \mathrm{Cl})$

\section{C}

Delayed Appropriate Therapy vs Appropriate Therapy Anderson et al [13] Daikos et al [11] Jung et al [16] Kim et al [19] Ortega et al [22] Direct meta-analysis
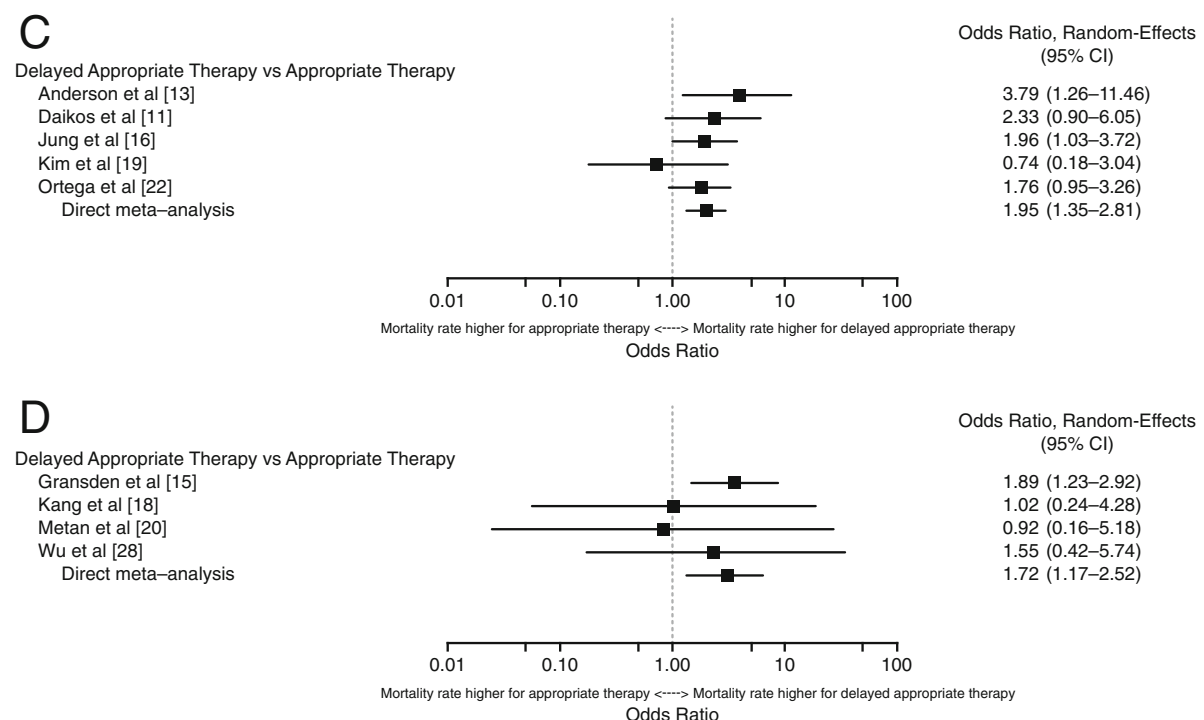

Fig. 3 Overall mortality for all studies with a DAT $>48 \mathrm{~h}$ or DAT $>$ C\& SR for infections due to (a) K. pneumoniae or E. coli, (b) K. pneumoniae alone, (c) K. pneumoniae with Tuon et al. [26] outlier removed, and (d) E. coli alone 
for DAT > C\& SR [11, 16, 22], Fig. 3b [11, 13, 16, 19, 22, 26] depicts the quantitative synthesis of these data, resulting in an OR of 1.60 (95\% CI, 0.97-2.64). Although the results suggest an increase in mortality associated with DAT $>48$ h or DAT $>$ C\& SR compared with early appropriate therapy, the increase was not statistically significant. Moderate heterogeneity existed $\left(\mathrm{I}^{2}=51.6 \%\right.$, $\mathrm{Qp}=0.092$ ) and was driven by the results in Tuon et al. [26], with an OR of 0.67. Tuon et al. was also 1 of 2 studies $[19,26]$ that showed a numeric trend of a higher rate of mortality in patients who received early appropriate therapy compared with those who received DAT > C\& SR. When a sensitivity analysis was conducted with Tuon et al. removed, the results became statistically significant, as shown in Fig. 3c [11, 13, 16, 19, 22] (OR, 1.95 [95\% CI, 1.35-2.81]), and there was no sign of heterogeneity $\left(\mathrm{I}^{2}=0.0 \%, \mathrm{Qp}=0.487\right)$.

Four studies $[15,18,20,28]$ described mortality associated with DAT $>48 \mathrm{~h}(n=3)$ or DAT $>$ C\& SR $(n=1)$ of $E$. coli infections. In these studies, DAT $>48 \mathrm{~h}$ or DAT $>$ C\& SR was associated with a significantly higher risk of overall mortality, with an OR of 1.72 (95\% CI, 1.17-2.52; Fig. 3d $[15,18,20,28])$. There was no sign of heterogeneity in the results $\left(\mathrm{I}^{2}=0.0 \%, \mathrm{Qp}=0.748\right)$.

\section{Delayed appropriate therapy $>24$ hours}

As shown in Table 2 [11-30], 5 studies described mortality associated with DAT $>24 \mathrm{~h}$ for either K. pneumoniae or E. coli infections [17, 21, 23, 24, 29]. There was a numeric trend suggesting a slight increase in mortality associated with DAT > 24h (OR, 1.24 [95\% CI, 0.851.80 ]; $\mathrm{I}^{2}=0.0 \%, \mathrm{Qp}=0.876$ ); however, these results were not statistically significant. For infections due to $K$. pneumoniae alone, there were no statistically significant differences for DAT $>24 \mathrm{~h} ; 3$ studies were included in the $>24-h$ analysis. Four studies described mortality associated with DAT $>24 \mathrm{~h}$ of $E$. coli infections alone. The OR of the meta-analysis was 1.19 (95\% CI, 0.73-1.93), a result that was not statistically significant. There was no sign of heterogeneity in the results $\left(\mathrm{I}^{2}=0, \mathrm{Qp}>0.20\right)$.

\section{Delayed appropriate therapy $>48$ hours}

Four studies described mortality associated with DAT $>$ $48 \mathrm{~h}$ of either K. pneumoniae or E. coli infections (Table 2) $[12,15,26,28]$. The meta-analyzed OR was 1.33 (95\% CI, 0.84-2.12), and although the point estimate suggests an increase in mortality associated with DAT $>48 \mathrm{~h}$, the result was not statistically significant. Heterogeneity was moderate $\left(\mathrm{I}^{2}=36.4, \mathrm{Qp}=0.146\right)$ and was driven by the more extreme results in Tuon et al. [26] $(\mathrm{OR}=0.67)$. For infections due to $K$. pneumoniae alone, there were insufficient data $(n=1)$ to conduct an analysis for DAT $>48 \mathrm{~h}$. Two studies $[15,28]$ described mortality associated with DAT $>48 \mathrm{~h}$ of $E$. coli infections alone. DAT $>48 \mathrm{~h}$ was associated with a significantly higher risk of overall mortality (OR, 1.86 [95\% CI, 1.23-2.80]). There was no sign of heterogeneity in the results $\left(\mathrm{I}^{2}=0.0 \%, \mathrm{Qp}=0.778\right)$.

\section{Delayed appropriate therapy $>72$ hours}

Four studies described mortality associated with DAT $>72$ $\mathrm{h}$ of either K. pneumoniae or E. coli infections (Table 2) [13, $18,19,25]$. The OR (2.12 [95\% CI, 0.66-6.79]) of the meta-analysis was not statistically significant. Heterogeneity was moderate $\left(\mathrm{I}^{2}=59.1 \%, \mathrm{Qp}=0.090\right)$ and was driven by the discrepancy of the results in Kim et al. [19] and Kang et al. [18] on the low end and Anderson et al. [13] and Schiappa et al. [25] on the high end. For infections due to $K$. pneumoniae alone, there were no statistically significant differences for DAT $>72 \mathrm{~h}$; only 2 studies reported analyzable data to contribute to the $>72-\mathrm{h}$ analysis. For infections due to $E$. coli alone, there were insufficient data $(n=1)$ to conduct an analysis for DAT $>72 \mathrm{~h}$.

\section{Delayed appropriate therapy $>C \& S R$}

Seven studies described mortality associated with DAT $>$ C\& SR of either K. pneumoniae or E. coli infections with an OR (95\% CI) of 2.06 (1.36-3.12; Table 2) $[11,14,16,20,22,27,30]$. Heterogeneity was moderate $\left(\mathrm{I}^{2}=33.5 \%\right.$, $\left.\mathrm{Qp}=0.211\right)$. For infections due to K. pneumoniae alone, DAT > C\& SR was associated with a significantly higher risk of mortality (OR, 1.93 [95\% CI, 1.29-2.89]). There was no sign of heterogeneity for this analysis $\left(\mathrm{I}^{2}=0.0 \%, \mathrm{Qp}=0.887\right)$. For infections due to $E$. coli alone, there were insufficient data $(\mathrm{n}=1)$ to conduct an analysis for DAT $>$ C\& SR.

\section{Discussion}

There were several notable findings from this systematic literature review and meta-analysis that predominately (95\%) included studies of patients with bloodstream infections due to $K$. pneumoniae or E. coli. First, the results suggest that overall, delays in appropriate therapy for patients with $K$. pneumoniae or E. coli infections result in higher mortality rates than when patients receive early appropriate therapy. On average, patients who receive any DAT (any delay exceeding $24 \mathrm{~h}$ after culture collection or C\& SR) have up to 2-fold higher odds of death. This is consistent with the observed association between time to appropriate therapy and mortality for serious infections due to other pathogens [31]. Likewise, the results of this meta-analysis indicate that a delay in appropriate therapy that exceeds $48 \mathrm{~h}$ or lasts until after the time of C\& SR is associated with an up to 2-fold higher risk of mortality. Taken together, the results for overall DAT suggest that even a delay of 24 to $48 \mathrm{~h}$ may negatively impact patient outcomes. Although not statistically significant, the ORs associated with DAT $>C \&$ SR only, which approximate a 48- to 72-h delay for 


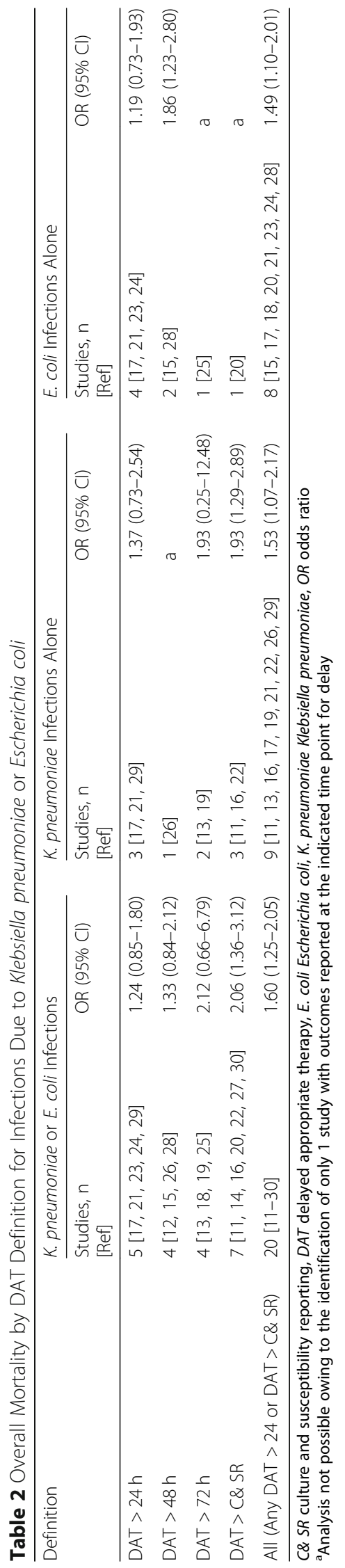


reporting of culture and susceptibility data, were highly consistent with the ORs for a $>48-$ to $>72-\mathrm{h}$ DAT. Finally, the results also suggest that the odds of mortality increased in a stepwise pattern as delays increased from $>24$ to $>72 \mathrm{~h}$. Although there were too few studies to draw definitive conclusions, it may be that the 24-h time point represents an inflection point beyond which a higher mortality risk is observed with increasing delays in initiating appropriate therapy. In general, the findings from this meta-analysis underscore the importance of timely initiation of appropriate therapy especially among patients with bloodstream infections due to K. pneumoniae or E. coli.

These findings have important implications for clinical practice, particularly highlighting the critical importance of using appropriate antibiotic therapy at initiation of treatment or "getting it right the first time." Delivery of early, appropriate therapy is a fundamental pillar of antibiotic stewardship, and delivery of an appropriate drug during the course of an infection is one of the most important measures clinicians can take for their patients to ensure the highest probability of a successful outcome. In particular, the findings suggest that clinicians should be more aggressive with their empiric antibiotic choices, especially among patients with bloodstream infections due to K. pneumoniae or E. coli. As part of this proposed practice shift, it is of the upmost importance that therapy is "de-escalated" once culture and susceptibility data are available, when possible. Otherwise, prolonged overuse of broad spectrum antibiotic will exacerbate our growing resistance problems and will limit the future utility of current antibiotics. Because the increasing prevalence of antibiotic resistance increases the likelihood of a patient receiving inappropriate empirical therapy, our findings also highlight the need to develop tools to both identify patients at high risk for antibiotic resistance and facilitate rapid diagnosis of the underlying causative pathogen to ensure early appropriate therapy.

Several things should be noted when interpreting the findings. Nineteen of the 20 studies included in this meta-analysis were of patients with bloodstream infections due to $K$. pneumoniae or $E$. coli. It is unclear whether the results are applicable to other infection types. Study design limitations included those inherent to any meta-analysis, such as heterogeneity across studies that may originate from differences in study populations, including those that combined both hospital- and community- onset infections, different definitions of response, different definitions of in vitro susceptibility and appropriateness, and the lack of a multivariate control. Heterogeneity was noted in several of the meta-analyses. Interestingly, the studies driving the heterogeneity were those that assessed the association between DAT and infections due to ESBLs. The definition of appropriate therapy varied across these studies and likely contributed to the observed heterogeneity. For example, the study by Tuon and colleagues [26] only considered carbapenems as appropriate for the treatment of infections due to ESBLs and classified all other beta lactam therapies as inappropriate; and resulted in a lower rate for appropriate therapy. However, cefepime and piperacillin/tazobactam are active against most ESBLs and therefore may have been considered appropriate therapy in other studies.

The use of a key objective endpoint (mortality) across all studies helped to minimize any bias. Nonetheless, the presence of publication bias cannot be excluded. In addition, a targeted analysis taking into account all confounders was not possible, limiting the ability of this meta-analysis to definitively establish the presence of a causal relationship. Because most of the included articles were retrospective observational cohort studies that were not randomized with regard to DAT, possible treatment selection bias and bias in management decisions with respect to dosage adjustments could not be analyzed or accounted for in the present analysis. It was also difficult to determine a definitive cutpoint for time delay given the limited number of studies included in this analysis. It is possible that there are different cutpoints for different infection types or disease severities. Another potential limitation is that the published literature included here focused predominantly on adults with bloodstream infections; pediatric and other infection types might differ in terms of the impact of DAT. Additional studies in these other infection types are necessary to explore this question. For example, Kumar and colleagues [32] reported that appropriate antibiotic therapy within the first hour is associated with improved survival in patients with septic shock. A final limitation is that this analysis did not evaluate other outcomes, such as length of hospital stay and costs. Data are needed to evaluate how delays in therapy affect these outcomes and whether there are different time delay cut-points.

\section{Conclusions}

In summary, results of this meta-analysis, which predominately (95\%) included studies of patients with bloodstream infections due to K. pneumoniae or E. coli, indicate that delayed appropriate therapy increases the risk of mortality, especially when appropriate treatment is delayed by $>48 \mathrm{~h}$. Given the results of this study documenting the adverse outcomes associated with delayed appropriate therapy, our findings suggest that there is a need to shift current treatment practices away from antibiotic escalation strategies that contribute to delayed appropriate therapy and toward early, relatively aggressive and comprehensive, antibiotic therapy, especially among patients with bloodstream infections due to $K$. 
pneumoniae or E. coli. As part of this proposed practice shift, it is critical that clinicians "streamline" therapy once culture and susceptibility data are available, when possible, to shorten the duration of time that patients unnecessarily receive broad spectrum antibiotics. Otherwise, prolonged overuse of broad spectrum antibiotics will exacerbate the growing resistance problem and will limit the future utility of current antibiotics. These findings also highlight the need for developing additional treatment options and strategies for infections in which therapeutic resistance is most likely to arise.

\section{Additional file}

Additional file 1: Search Algorithms. (PDF 260 kb)

\begin{abstract}
Abbreviations
C\& SR: Culture and susceptibility reporting; CAZ-R: Ceftazidime-resistant: CRE: Carbapenem-resistant Enterobacteriaceae; CTX-R: Cefotaxime resistant; DAT: Delayed appropriate therapy; E. coli: Escherichia coli; ESBL: Extendedspectrum beta lactamase; K. pneumoniae: Klebsiella pneumoniae; KPC: K. pneumoniae carbapenemase; NR: Not reported; OR: Odds ratio; PICOST: Participants, interventions, comparisons, outcomes, study design, and time period; PRISMA: Preferred Reporting Items for Systematic Reviews and MetaAnalyses; UTI: Urinary tract infection; VPKP: VIM-1-producing Klebsiella pneumoniae
\end{abstract}

\section{Acknowledgments}

Writing and editorial assistance was provided to the authors by Bret Fulton, RPh, and Kevin Ryder, PhD, of Complete Healthcare Communications, LLC (North Wales, PA). All authors met the International Committee of Medical Journal Editors authorship criteria. Neither honoraria nor payments were made for authorship.

\section{Funding}

This work was supported by Allergan plc (Dublin, Ireland), as was the writing and editorial assistance. Allergan played a role in the study design; in the collection, analysis, and interpretation of the data; in the writing of the report; and in the decision to submit the article for publication.

\section{Availability of data and materials}

The datasets used and/or analysed during the current study are available from the corresponding author on reasonable request.

\section{Authors' contributions}

Study design: TPL, QZ, KF, AM. Collection, analysis, and/or interpretation of the data: TPL, QZ, KF, PJG, AM. Writing of the report: TPL, QZ, KF, PJG, AM. Decision to submit the article for publication: TPL, QZ, KF, PJG, AM. All authors read and approved the final manuscript.

\section{Ethics approval and consent to participate} Not applicable.

\section{Consent for publication}

Not applicable.

\section{Competing interests}

TPL has received consulting fees or honoraria and support for travel to meetings for this study or other purposes from Allergan plc. He has also been a consultant for Merck and The Medicines Company and has received payment for lectures including service on speakers bureaus for Allergan for work not associated with the current study. QZ was an employee of Allergan at the time of study conduct and analysis. KF and AM are employees of Evidera. Evidera received payment for conducting the analyses on which this study is based. PJG is an employee of Allergan and may hold stock or stock options.

\section{Publisher's Note}

Springer Nature remains neutral with regard to jurisdictional claims in published maps and institutional affiliations.

\section{Author details}

${ }^{1}$ Department of Pharmacy Practice, Albany College of Pharmacy and Health Sciences, Albany, NY, USA. ${ }^{2}$ Global Health Economics and Outcomes Research, Allergan plc, Madison, NJ, USA. ${ }^{3}$ Meta Research, Evidera, Bethesda, MD, USA. ${ }^{4}$ Global Health Economics and Outcomes Research, Allergan plc, 2525 Dupont Drive, Irvine, CA 92612, USA.

Received: 9 August 2018 Accepted: 19 November 2018

Published online: 05 December 2018

\section{References}

1. Hawser SP, Badal RE, Bouchillon SK, Hoban DJ, Hackel MA, Biedenbach DJ, Goff DA. Susceptibility of gram-negative aerobic bacilli from intraabdominal pathogens to antimicrobial agents collected in the United States during 2011. J Inf Secur. 2013;68:71-6.

2. Zilberberg MD, Shorr AF. Secular trends in gram-negative resistance among urinary tract infection hospitalizations in the United States, 2000-2009. Infect Control Hosp Epidemiol. 2013;34:940-6.

3. Centers for Disease Control and Prevention. Antibiotic resistance threats in the United States, 2013. Available at: http://www.cdc.gov/drugresistance/ threat-report-2013/pdf/ar-threats-2013-508.pdf. Accessed Sept 12, 2018.

4. Micek ST, Heard KM, Gowan M, Kollef MH. Identifying critically ill patients at risk for inappropriate antibiotic therapy: a pilot study of a point-of-care decision support alert. Crit Care Med. 2014;42(8):1832-8.

5. Micek ST, Reichley RM, Kollef MH. Health care-associated pneumonia (HCAP): empiric antibiotics targeting methicillin-resistant Staphylococcus aureus (MRSA) and Pseudomonas aeruginosa predict optimal outcome. Medicine. 2011;90:390-5.

6. Cosgrove SE. The relationship between antimicrobial resistance and patient outcomes: mortality, length of hospital stay and health care costs. Clin Infect Dis. 2006;42(suppl 2):S82-9.

7. Tzouvelekis LS, Markogiannakis A, Piperaki E, Souli M, Daikos GL. Treating infections caused by carbapenemase-producing Enterobacteriaceae. Clin Microbiol Infect. 2012;20:862-72.

8. Vardakas KZ, Tansarli GS, Rafailidis PI, Falagas ME. Carbapenems versus alternative antibiotics for the treatment of bacteraemia due to Enterobacteriaceae producing extended-spectrum beta-lactamases: a systematic review and meta-analysis. J Antimicrob Chemother. 2012;67: 2793-803.

9. R Core Team. R: A language and environment for statistical computing. $R$ Foundation for Statistical Computing. Available at: https://www.R-project. org. Accessed Feb 24, 2016.

10. Thompson SG. Why sources of heterogeneity in meta-analysis should be investigated. BMJ. 1994;309:1351-5.

11. Daikos GL, Petrikkos P, Psichogiou M, Kosmidis C, Vryonis E, Skoutelis A, Georgousi K, Tzouvelekis LS, Tassios PT, Bamia C, et al. Prospective observational study of the impact of VIM-1 metallo-beta-lactamase on the outcome of patients with Klebsiella pneumoniae bloodstream infections. Antimicrob Agents Chemother. 2009;53:1868-73.

12. Abhilash KP, Veeraraghavan B, Abraham OC. Epidemiology and outcome of bacteremia caused by extended spectrum beta-lactamase-producing Escherichia coli and Klebsiella spp. in a tertiary care teaching hospital in South India. J Assoc Physicians India. 2010;58:13-7.

13. Anderson DJ, Engemann JJ, Harrell LJ, Carmeli Y, Reller LB, Kaye KS. Predictors of mortality in patients with bloodstream infection due to ceftazidime-resistant Klebsiella pneumoniae. Antimicrob Agents Chemother. 2006;50:1715-20.

14. Du B, Long Y, Liu H, Chen D, Liu D, Xu Y, Xie X. Extended-spectrum betalactamase-producing Escherichia coli and Klebsiella pneumoniae bloodstream infection: risk factors and clinical outcome. Intensive Care Med. 2002;28: 1718-23.

15. Gransden WR, Eykyn SJ, Phillips I, Rowe B. Bacteremia due to Escherichia coli: a study of 861 episodes. Rev Infect Dis. 1990;12:1008-18.

16. Jung $Y$, Lee MJ, Sin HY, Kim NH, Hwang JH, Park J, Choe PG, Park WB, Kim ES, Park SW, et al. Differences in characteristics between healthcare-associated and community-acquired infection in community- 
onset Klebsiella pneumoniae bloodstream infection in Korea. BMC Infect Dis. 2012;12:239.

17. Kang Cl, Kim SH, Wan BP, Lee KD, Kim HB, Kim EC, Oh MD, Choe KW. Bloodstream infections due to extended-spectrum (beta)-lactamaseproducing Escherichia coli and Klebsiella pneumoniae: risk factors for mortality and treatment outcome, with special emphasis on antimicrobial therapy. Antimicrob Agents Chemother. 2004;48:4574-81.

18. Kang Cl, Wi YM, Ko KS, Chung DR, Peck KR, Lee NY, Song JH. Outcomes and risk factors for mortality in community-onset bacteremia caused by extended-spectrum beta-lactamase-producing Escherichia coli, with a special emphasis on antimicrobial therapy. Scand J Infect Dis. 2013;45:519-25.

19. Kim BN, Woo JH, Kim MN, Ryu J, Kim YS. Clinical implications of extendedspectrum (beta)-lactamase-producing Klebsiella pneumoniae bacteraemia. J Hosp Infect. 2002;52:99-106.

20. Metan G, Zarakolu P, Cakir B, Hascelik G, Uzun O. Clinical outcomes and therapeutic options of bloodstream infections caused by extendedspectrum (beta)-lactamase-producing Escherichia coli. Int J Antimicrob Agents. 2005;26:254-7.

21. Micek ST, Welch EC, Khan J, Pervez M, Doherty JA, Reichley RM, Kollef MH. Empiric combination antibiotic therapy is associated with improved outcome against sepsis due to gram-negative bacteria: a retrospective analysis. Antimicrob Agents Chemother. 2010;54:1742-8.

22. Ortega M, Marco F, Soriano A, Almela M, Martinez JA, Lopez J, Pitart C, Mensa J. Cefotaxime resistance and outcome of Klebsiella spp bloodstream infection. Eur J Clin Microbiol Infect Dis. 2011:30:1599-605.

23. Park SH, Choi SM, Lee DG, Kim J, Choi JH, Kim SH, Kwon JC, Yoo JH. Emergence of extended-spectrum (beta)-lactamase-producing Escherichia coli as a cause of community-onset bacteremia in South Korea: risk factors and clinical outcomes. Microb Drug Resist. 2011;17:537-44.

24. Rodriguez-Bano J, Picon E, Gijon P, Hernandez JR, Cisneros JM, Pena C, Almela M, Almirante B, Grill F, Colomina J, et al. Risk factors and prognosis of nosocomial bloodstream infections caused by extendedspectrum-(beta)-lactamase-producing Escherichia coli. J Clin Microbiol. 2010;48:1726-31.

25. Schiappa DA, Hayden MK, Matushek MG, Hashemi FN, Sullivan J, Smith KY, Miyashiro D, Quinn JP, Weinstein RA, Trenholme GM. Ceftazidime-resistant Klebsiella pneumoniae and Escherichia coli bloodstream infection: a casecontrol and molecular epidemiologic investigation. J Infect Dis. 1996;174: $529-36$

26. Tuon FF, Kruger M, Terreri M, Penteado-Filho SR, Gortz L. Klebsiella ESBL bacteremia-mortality and risk factors. Braz J Infect Dis. 2011;15:594-8.

27. Wang SS, Lee NY, Hsueh PR, Huang WH, Tsui KC, Lee HC, Wu CJ, Chang CM, Huang CC, Huang CF, et al. Clinical manifestations and prognostic factors in cancer patients with bacteremia due to extended-spectrum $\beta$-lactamaseproducing Escherichia coli or Klebsiella pneumoniae. J Microbiol Immunol Infect. 2011;44:282-8.

28. Wu UI, Chen WC, Yang CS, Wang JL, Hu FC, Chang SC, Chen YC. Ertapenem in the treatment of bacteremia caused by extended-spectrum betalactamase-producing Escherichia coll: a propensity score analysis. Int J Infect Dis. 2012;16:e47-52.

29. Zarkotou O, Pournaras S, Tselioti P, Dragoumanos V, Pitiriga V, Ranellou K, Prekates A, Themeli-Digalaki K, Tsakris A. Predictors of mortality in patients with bloodstream infections caused by KPC-producing Klebsiella pneumoniae and impact of appropriate antimicrobial treatment. Clin Microbiol Infect. 2011;17:1798-803.

30. Lee NY, Lee CC, Huang WH, Tsui KC, Hsueh PR, Ko WC. Carbapenem therapy for bacteremia due to extended-spectrum- $\beta$-lactamase-producing Escherichia coli or Klebsiella pneumoniae: implications of ertapenem susceptibility. Antimicrob Agents Chemother. 2012;56:2888-93.

31. Park JH, Choi SH, Chung JW. The impact of early adequate antimicrobial therapy on 14-day mortality in patients with monomicrobial Pseudomonas aeruginosa and Acinetobacter baumannii bacteremia. J Infect Chemother. 2013;19:843-9.

32. Kumar A, Roberts D, Wood KE, Light G, Parrillo JE, Sharma S, Stuppes R, Feinstein D, Zanotti S, Talberg $L$, et al. Duration of hypotension before initiation of effective antimicrobial therapy is the critical determinant of survival in human septic shock. Crit Care Med. 2006;34:1589-96.

Ready to submit your research? Choose BMC and benefit from:

- fast, convenient online submission

- thorough peer review by experienced researchers in your field

- rapid publication on acceptance

- support for research data, including large and complex data types

- gold Open Access which fosters wider collaboration and increased citations

- maximum visibility for your research: over $100 \mathrm{M}$ website views per year

At BMC, research is always in progress.

Learn more biomedcentral.com/submissions 